\title{
FORMATION CONTROL AND ROLE ASSIGNMENT OF AUTONOMOUS MOBILE ROBOTS IN UNSTRUCTURED ENVIRONMENT
}

\author{
Fatima Debbat* and Lounis Adouane**
}

\begin{abstract}
Coordinating distributed robots to work in formation becomes one of the complex problems in multi-robot systems during the last years. The main contribution in the proposed work is to control non-holonomic mobile robots to maintaining a desired formation during navigation and the way to change formation shape when the robots detect a hindering obstacle. The robots are anonymous and randomly distributed. The developed control strategy, modeled using the Petri Nets formalism, combines together formation control, navigation and obstacle avoidance. The formation control is based on a leader-follower formation approach and the obstacle avoidance is based on modified limit-cycle method [1]. The overall control architecture is validated through a multitude of simulation.
\end{abstract}

\section{Key Words}

Mobile robot, formation control, leader-follower approach, role switching, obstacle avoidance, limit-cycle method

\section{Introduction}

Currently, navigation in formation of a group of mobile robots receives a very great interest by multi-robot community. Various advantages have been shown in several application compared with a single mobile robot [2], [3], [4], [5], [6]. In this paper, we focus on the problem of how to control a group of mobile robots moving in formation in an unknown environment and while switching from a specific formation to another. We propose a resolution strategy of initialization, maintaining formation, and switching in other shape when perceptual event occurs. An improvement of an obstacle avoidance approach using limit-cycle [1], [7], [8] was proposed. This approach is characterized by its simplicity, no need for global knowledge and allows

\footnotetext{
* Faculty of Sciences and Technology, University of Mascara, Mascara, Algeria; e-mail: debbatfatima@gmail.com

** Institut Pascal, Blaise Pascal University, Clermont-Ferrand, France; e-mail: Lounis.Adouane@univ-bpclermont.fr
}

to choose the direction of avoidance according to the target position. For this purpose, we design an adaptive controller to drive a group of mobile robots in a leader-follower configuration. This control allows the robots group: to explore the environment, to initialize a formation shape, to change the formation structure according to the navigation context, and to avoid obstacles. Petri nets formalism is used to modelling the overall control architecture embedded in each robot.

\section{Navigation Control Strategy of the Group of Robot}

This study proposes a Petri Net [9] model in order to managing the different states allowed for the formation control. The total functionality of the group is decomposed into functional behaviours. The proposed net (cf. Fig. 1) comprises 6 places and 12 transitions.

\subsection{Formation Initialization}

The mobile robots must arrange themselves into a specific geometric configuration without centralized control. Only information from local sensors are used which are inevitably incomplete. Initially (cf. Fig. 1), the robots are randomly positioned in the environment. The robots begin to explore and observe the environment in order to find other robots while avoiding the detected static obstacles (P1: Exploration). Each robot will be in P2 (Move state) if it detects another robot. It tries to join the other robot until certain distance (to do not collide with it) while avoiding detected obstacle in its way. When the robot is close enough to the other robot, it will be in P3 (Position Assignment state).

\subsection{Obstacle Avoidance}

The mobile robots considered in this study are wheeled mobile robots of unicycle type. The obstacle avoidance is enabled if the distance between robot and obstacle is smaller than a specific distance and the obstacle is located 


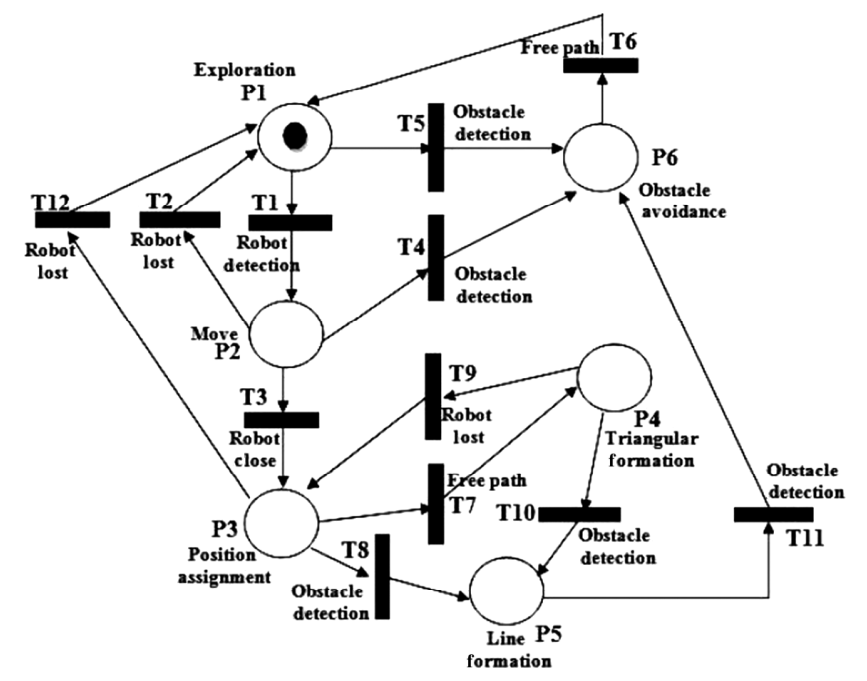

Figure 1. Petri Net for the proposed formation control (embedded in each robot).

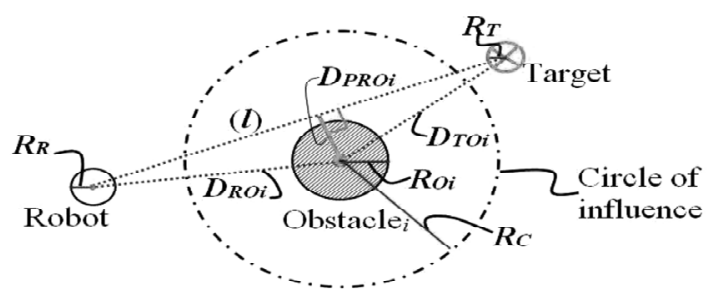

Figure 2. The used perceptions for mobile robot navigation [1].

on the robot way. A real-time obstacle avoidance method named limit-cycle method [1] has been implemented. One supposes in the setup that obstacles and the robot could surround by bounding cylindrical boxes with respectively $\boldsymbol{R}_{\boldsymbol{O}}$ and $\boldsymbol{R}_{\boldsymbol{R}}$ radii. The target to reach is also characterized by a circle of $\boldsymbol{R}_{T}$ radius. Several perceptions are also necessary for the robot navigation: $D_{R O i}$ distance between the robot and the obstacle " $i$," $D_{P R O i}$ perpendicular distance between the line $(l)$ and the obstacle " $i$," and $D_{T O i}$ distance between the target and the obstacle " $i$ " (Fig. 2).

For each detected obstacle we define a circle of infiuence with a radius of $R c=R_{R}+R_{O i}+$ Margin. Margin corresponds to a safety tolerance.

The limit-cycle method in its original version assumes that the obstacles have a known surface and a geometrical shape, but in an unstructured environment this knowledge is not always available to the robots. In this context, a solution for obstacles whose shape is unknown is proposed. We follow the same limit-cycle algorithm but instead of surrounding the obstacle by the circle of influence, this circle surround only the first detected point of this obstacle (cf. Fig. 3).

The circle radius represents the distance between the robot and the obstacle and equals $R_{R}+$ Margin. We repeat this process each $T$ equals to $\frac{R_{R}}{v_{R}}$ with $v_{R}$ is the robot velocity.

\subsection{Navigation in Formation}

In this section, we will develop and describe the role assignment between robots and how robots choose the appropriate formation. The proposed strategy is composed by different states (cf. Fig. 1). A leader-follower approach is used for the initialization of mobile formations [10]. Initially, each robot is considered as leader. When it detects another robot, it remains leader according to the priority given by its identification number (ID). In P3 position assignment (cf. Fig. 1) an information exchange on the ID value between the robots is necessary for the recruitment of the leader. Each robot has its identification number, called Robot ID (RID). The RID determines the priority of

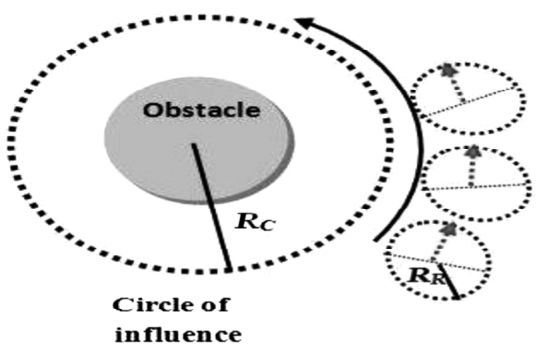

(a)

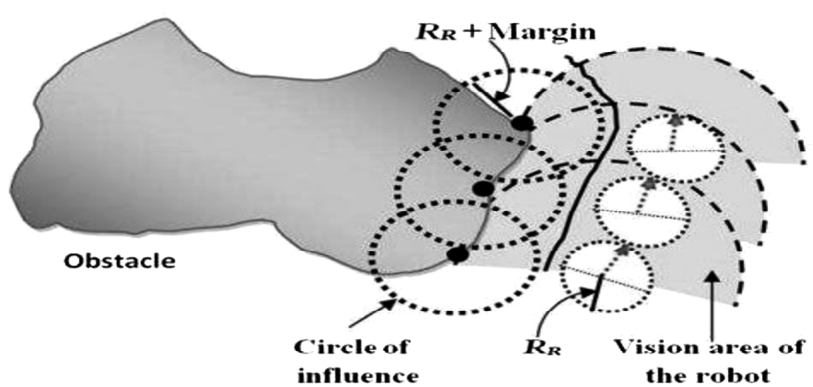

(b)

Figure 3. Avoidance of the obstacle using limit-cycle method: (a) known obstacle shape and (b) unknown obstacle shape. 


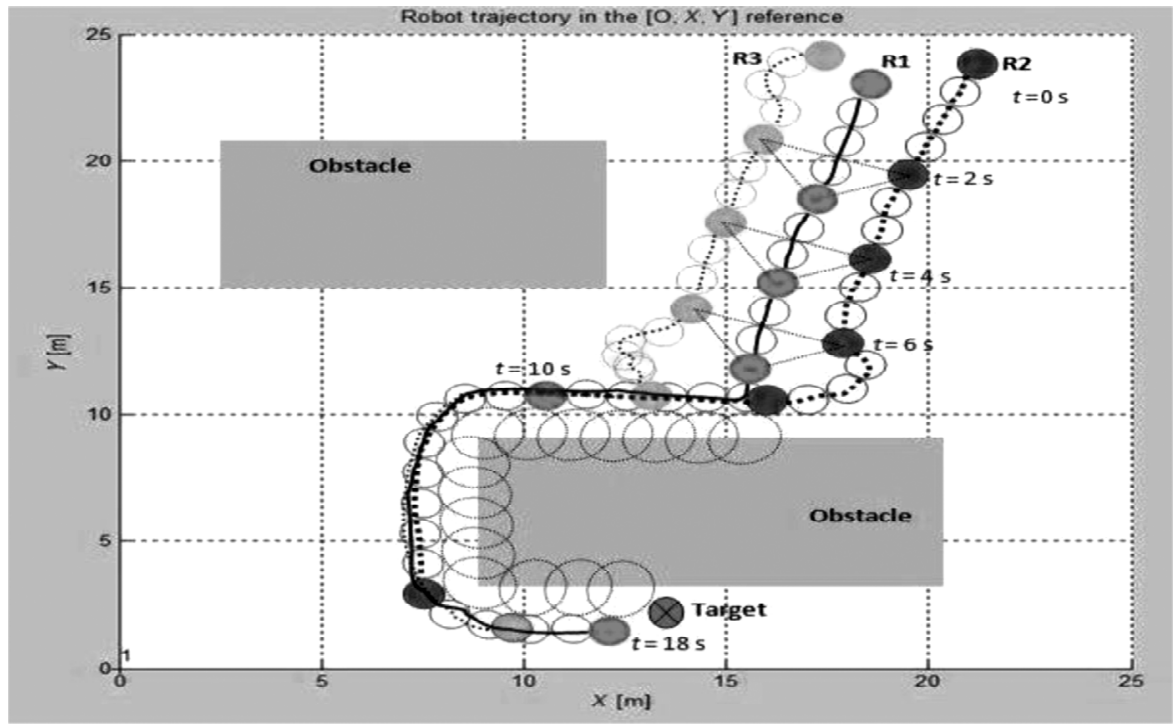

Figure 4. Navigation with keeping and switching of the formation shape.

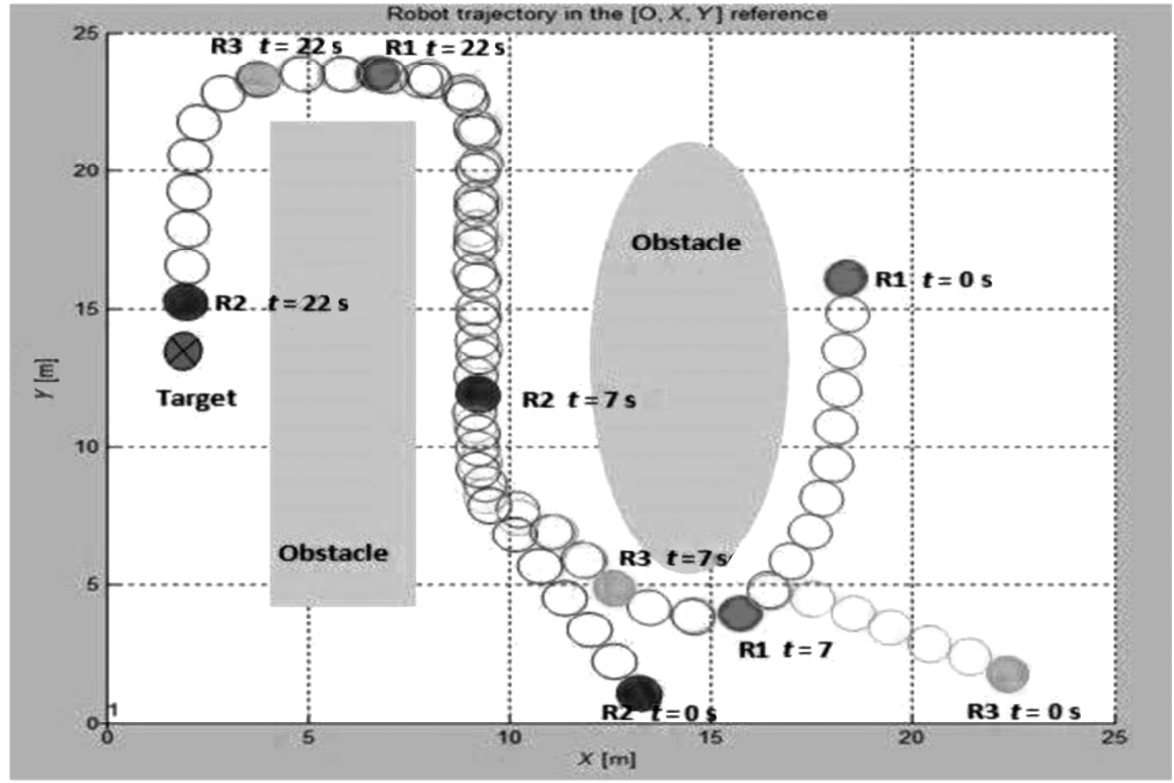

Figure 5. Navigating in formation while avoiding obstacles.

a robot in formation. In the proposed strategy, the RID of the leader robot is the smallest. In this work, we adopted two types of formation shape: triangular formation and line formation. The switching mechanism between them is given by following the proposed process:

1. If the path is free (T7), the robots organize themselves according to the triangular formation. This shape is realized by using $(L-L)$ control [10].

2. If an obstacle is detected and the robots are in Triangle formation (P4), the robots organize themselves according to the line formation in order to ensure a safe avoidance (T10). This shape is realized by using $(\psi-L)$ control [10].

3 . If one of the robots does not see any of other robots, it returns to P1 (Exploration state).
4. If an obstacle is detected during assignment position process, the robots organize themselves according to the line formation (T8).

\section{Simulation Results}

Examples of simulation results are given in Figs. 4 and 5 . The plots correspond to the simulation results of the robot team navigating in cluttered environment. The initial positions of three robots are randomly generated and we want to keep the relative distance and angle between two consecutive robots as constant fixed value: $d_{L}=2 \mathrm{~m}$, and $\psi_{L}=0^{\circ}$ in Line formation and Triangle formation. $\left(d_{L}\right.$ : distance between leader and follower, $\psi_{L}$ : angle between leader and follower). The distance between followers is $d_{F}=2 \sqrt{ } 2 \mathrm{~m}$ in Triangle formation and $d_{F}=2 \mathrm{~m}$ in Line 
formation. Figure 4 shows the trajectory of three robots in the second simulation case. The robots navigate in formation (triangular shape) in order to reach the target. When an obstacle is detected, the robots activate the avoidance obstacle behaviour while changing the formation shape to line.

Other ability of the proposed control architecture is observed in Fig. 5. In this case, the three robots try to join a target. When the robots are close to the obstacle, robot $\mathrm{R} 2$ relinquishes the leadership to the robot R3 (Formation switching behaviour).

\section{Conclusion}

In this paper, it is presented a new decentralized formation control for small-scale mobile robot team using the leaderfollower strategy. The proposed control resolves the problems of obstacle avoidance and formations changes. In the proposed approach, the autonomous mobile robots must arrange themselves into a specific geometric configuration without centralized control. The approach has been tested through a multitude of simulations to demonstrate validity and effectiveness of the proposed control architecture. The future research work will focus on extending the results to more general applications by employing more than three robots in formation.

\section{References}

[1] L. Adouane. Orbital obstacle avoidance algorithm for reliable and on-line mobile robot navigation, 9th Conference on Autonomous Robot Systems and Competitions, May 2009.

[2] G. Lozenguez, L. Adouane, A. Beynier, P. Martinet, and A.-I. Mouaddib, Map partitioning to approximate an exploration strategy in mobile robotics, Advances on practical applications of agents and multi-agent systems, (Springer Berlin Heidelberg, 2011), 63-72.

[3] M.T. Khan, Izhar, F. Nasir, M.U. Qadir, and C.W. de Silva, Multi-robot cooperation framework based on artificial immune system, Control and Intelligent Systems, 43(3), 2015, 2705.

[4] L. Geng, Y.F. Zhang, J.J. Wang, J.Y.H. Fuh, and S.H. Teo, Cooperative task planning for multiple unmanned aerial vehicles using a genetic algorithm, Control and Intelligent Systems, 201(2), 2014, 2562.

[5] A. Benzerrouk, L. Adouane, P. Martinet, and N. Andreff, Toward an hybrid control architecture for a mobile multirobot systems, CAR'08, 3rd National Conference on Control Architectures of Robots, 2008.

[6] A. Benzerrouk, L. Adouane, L. Lequievre, and P. Martinet, Navigation of multi-robot formation in unstructured environment using dynamical virtual structures, IROS 10, IEEE/RSJ International Conference on Intelligent Robots and Systems, Taipei-Taiwan, 2010.
[7] M.S. Jie, J.H. Baek, Y.S. Hong, and K.W. Lee, Real time obstacle avoidance for mobile robot using limit-cycle and vector field method, Knowledge-Based Intelligent Information and Engineering Systems, October 2006, 866-873.

[8] J.M Vilca, L. Adouane, and Y. Mezouar, Reactive navigation of mobile robot using elliptic trajectories and effective online obstacle detection, Gyroscopy and Navigation, 4(1), 2013, $14-25$.

[9] T. Murata, Petri nets: properties, analysis and applications, Proc IEEE, 77(4), 1989, 541-580.

[10] L. Consolini, F. Morbidi, D. Domenico, and M. Tosques. Leader follower formation control of nonholonomic mobile robots with input constraints, Automatica, 44, 2008, 1343-1349.

\section{Biographies}

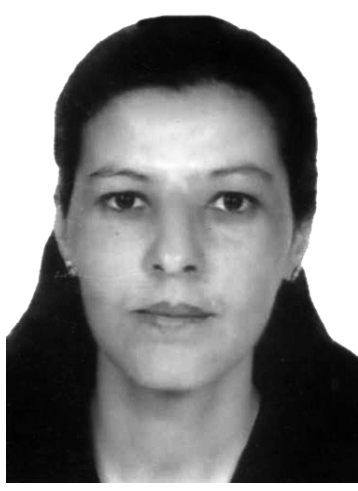

Fatima Debbat received her Master of Sciences in Space Technologies in 2002 from the Space Techniques Center (CTS) Algeria, and Ph.D. in Electronics in 2007 from the University of Tlemcen, Algeria. She is currently Associate Professor in the Department of computer Science at Mascara University, Algeria. Her research interests include Artificial Intelligence Applications, Optimization, Mobile Robotics Control, and Wireless Networks.

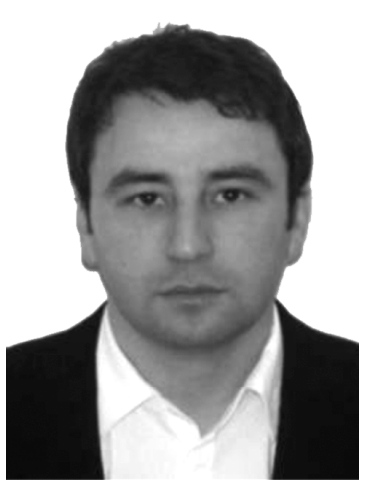

Lounis Adouane received his Master of Sciences in 2001 from IRCCyN - ECN Nantes (France), where he worked on the control of legged mobile robotics. In 2005 he obtained his Ph.D. in Automatic Control from LAB UFC Besançon. During his Ph.D. Adouane has deeply investigated the field of multi-robot systems, especially those relaying to reactive control architectures. After that, he joined, in 2006, LAI - INSA Lyon and he studied the hybrid architecture of control applied to cooperative mobile arms robots. Since 2006, he is Associate Professor at Institut Pascal - Polytech Clermont-Ferrand. His research interests include Mobile Robotics Control, Cooperative Robotics, Artificial Intelligence, Behavioral/Hybrid Control Architectures, and Multi-Robot Simulation. 